\title{
Resources for optics in middle school education
}

\section{Stephen Pompea, Michael Nofziger}

Stephen M. Pompea, Michael J. Nofziger, "Resources for optics in middle school education," Proc. SPIE 2525, 1995 International Conference on Education in Optics, (13 October 1995); doi: 10.1117/12.224015

SDIE Event: SPIE's 1995 International Symposium on Optical Science, Engineering, and Instrumentation, 1995, San Diego, CA, United States 
Resources for optics in middle school education

\author{
Stephen M. Pompea ${ }^{1}$ \\ Pompea \& Associates \\ 1321 East Tenth Street \\ Tucson, Arizona 85719-5808 \\ Electronic mail: spompea@as.arizona.edu \\ Michael J. Nofziger \\ Optical Sciences Center \\ University of Arizona \\ Tucson, AZ 8572 \\ Electronic mail: nofziger@gas.uug.arizona.edu
}

\begin{abstract}
This paper describes some of our experiences in Tucson in support of optics education in the middle school. A workshop for middle school teachers and work with middle school classes has identified a number of approaches that may be effective for the integration of optics into the middle school science curriculum.
\end{abstract}

Keywords: optics education, middle school, demonstrations, teacher training, curriculum planning

\title{
1. INTRODUCTION
}

Optics is not a normal part of the middle school curriculum. However, it is an area that is quite exciting to students and teachers (Pompea, 1994). Our experience with middle school classes and teachers has made us aware of a number of areas where optics can be integrated into the middle school curriculum Teachers at middle schools are looking for curriculum materials and for assistance from the optics community. A number of projects are underway in Tucson that may serve as prototypes for the development of materials and curriculum for integrating optics into the middle school. This paper primarily explores some of the specific topics that have been successfully treated at the middle school level.

\section{HANDS-ON EXPERIMENTS}

The experiences of the authors in working with middle school students is that hands-on experiments are particularly effective. Problem-centered learning is also effective since many students greatly enjoy the challenge of solving problems. Demonstrating an optical phenomena and then letting the students explain it is interesting for many students. For example a student can be given a piece of non-glare glass (which is frosted on one side) and is then asked which side should be facing outwards in a picture frame. This question will provoke discussion and experimentation among students. It also gives students a chance to test their reasoning ability and to interact with other students. Students also enjoy manipulating lenses, holograms, laser pointers, fiber optics, and other tangible objects. We have based many of our activities on the use of hands-on experiments because the students enjoy them so much.

\section{TOPICS FOR OPTICS TEACHING}

Some of the topics which are of greatest interest to students of this age group are the following.

\footnotetext{
1 Adjunct Associate Astronomer, Steward Observatory, University of Arizona 85721 Voice/Fax (520) 792-2366/622-2122
} 


\begin{tabular}{|l|l|l|}
\hline Optical communication & Lasers & Optics of microscopes and telescopes \\
\hline Holograms & Black Lights & Diffraction gratings \\
\hline Scattering & Reflection & Sky optics \\
\hline Optics and special effects & Optical Illusions & Image formation \\
\hline
\end{tabular}

The gateway to most of these topics is a piece of optical equipment to demonstrate the phenomena. We are prototyping an optics kit which has some items which are somewhat different than the Optical Society of America Optical Kit. This effort is not meant to supplant the kit but rather to create a kit that is more closely suited to the kinds of experiments we want to use in Tucson with students.

Many of the students have some access to computers and even to the Internet. To support computer-based instruction we are prototyping a few computer animations of optical phenomena to see what kinds of misconceptions students have about optical phenomena. For example, an animation about how light scatters off surfaces of different roughnesses will be used to explore student conceptions and misconceptions about scattering.

\section{SPECIFIC IDEAS FOR TEACHING OPTICS}

In this section we discuss various ideas for teaching optics at the middle school level. An underlying theme is to use relatively inexpensive materials, and practical examples from everyday experience. The goal is to teach fundamental principles of optics, at the same time motivating the students to further explore optics in the world around them. Note that some of these ideas are also discussed in the paper "Optics curriculum for middle school students" which appears elsewhere in this volume.

\section{A. Colors of the Visible Spectrum}

Traditionally, the study of optics begins with a look at the entire electromagnetic spectrum and the relatively narrow range of wavelengths that the human eye can see. Experience has shown that this is a good starting point for students at the middle school level, as well. If one can obtain transmission filters for the red, blue, and green primary colors, additive color mixing may be demonstrated. If filters for their complimentary colors can be obtained, subtractive color mixing may be demonstrated. This would be very useful as an introduction to the use of a microscope to observe the dot pattern of complimentary colors used in color half-tone printing.

Another demonstration that captures the imagination of the students is to project a large-scale rainbow on the wall of the classroom. This may be done using a combination of a prism and a mirror, held in front of an overhead projector (see the paper referenced above for more details). If one is lucky enough to have a pair of laser goggles available, students may see firsthand the processes of absorption and spectral filtering. (Glass filters work as well, but don't have the "imaginative" impact on the students that laser goggles do!)

\section{B. Sources of Light}

The discussion of various sources of light is both practical and common to students' everyday experience. It is useful to have them think about light sources, instead of using the typical lecture approach. They should quickly be able to list naturally-ocurring sources (fire, the sun, stars, sunlight reflected from the moon, lightning, and the rainbow), as well as man-made sources (tungsten and fluorescent bulbs, "neon" signs, LED's, and lasers). Sources that are not so obvious include fireflies, heater elements (such as in a toaster), and remote controls, to name but a few.

- Activity: Have the students keep a list of sources they discover throughout the duration of the unit on optics. 


\section{Spectra}

It is instructive and fun for the students to be able to actually see the spectra of the light sources they discover. A small piece of plastic diffraction grating held in a cardboard frame becomes such a "tool." It is recommended that a high-quality (flexible plastic) grating having no more than $500-600$ lines $/ \mathrm{mm}$ be used.

- Activity: Investigate the spectra from tungsten bulbs, fluorescent bulbs, LED's, streetlights, "neon" signs, and lightning (under controlled classroom conditions, have them look at reflected laser light as well). What do they observe as a sodium street lamp warms up? Can they resolve the orange doublet line in a mercury street lamp?

\section{Plane Mirrors}

The study of plane mirrors is fundamental to any treatment of optics. Introduce the law of reflection and the concept of handedness, or parity. Have the students observe this by looking at the reflection of their right hand in an actual mirror. Get them to realize that this is why the word "ambulance" is written backwards on the front of that vehicle.

Talk about the concept of reflectivity, and discuss why a back-surface mirror actually produces two reflections. Again, have them observe this for themselves. It is always interesting to learn that a transparent piece of glass becomes a very good mirror when looked across at glancing incidence.

- Activity: Have the students use two mirrors to build a simple periscope. Use the concepts of the law of reflection and reflectivity to explain how an car's rear-view mirror works.

\section{E. Curved Mirrors}

A discussion of how curved mirrors work is somewhat difficult for students at this level, since it involves the concept of ray-tracing. Also, curved mirrors are more expensive to obtain than plane mirrors. Still, it is easy enough to introduce the concept of center of curvature and focal point for a concave mirror. Have the students observe what happens when they position the pupil of their eye at the center of curvature. A common example of a convex mirror is the side mirror found on the sides of most automobiles.

- Activity: Have the students examine what the image looks like for an object placed inside of the center of curvature of a concave mirror? What does it look like if it is placed outside of the center of curvature? What do images look like using convex mirrors?

\section{F. Lenses}

The study of lenses is always fun and challenging for students at this level. A lens may be modeled as a successive stack of prisms, with a plane parallel plate in the center. By grinding and polishing the discontinuities, a smooth lens emerges. A positive lens is one that is thicker in the middle than at the edges, and vice versa for a negative lens. Draw various possible shapes--plano-concave, bi-concave, plano-convex, and bi-convex.

Provide each of them with a collection of cheap glass (or plastic) positive and negative lenses. Ask them to separate the positive lenses from the negative lenses based on their shapes. (For this exercise, it is most useful to obtain surplus lenses not all of the same size or shape.)

\section{G. Image Formation}

The concept of image formation is fundamental to the understanding of basic optics. A discussion of real vs. virtual images is needed to begin this part (the images seen in the plane mirrors are examples of virtual images). 
Simple ray tracing could be used to show how real and virtual images are formed, but hands-on observation are also instructive. The collection of lenses just looked at can be used to: (a) form a real image using a positive lens, and measure the focal length of the lens, (b) form a virtual image using a positive lens, i.e., a simple magnifier, and (c) form a virtual image using a negative lens. It should be pointed out that a negative lens forms only a virtual image. Furthermore, make sure students understand and prove for themselves that the orientation of the lenses doesn't effect the orientation of the images formed. Students are always thrilled to actually see a positive lens form a real image that is inverted.

One interesting and fundamental set of experiments really probes students' understanding of how lenses work. Obtain two sets, of two positive lenses each, for every student. The first set has lenses of the same diameter but different focal lengths. Have the students investigate the difference in the size of the images produced. The second set has lenses of the same focal length, but quite different diameters. Have students discover which image is brighter. Of course, the images produced by the first set of lenses will also be of different brightness, but for a different reason, because the same amount of light through each lens is spread over different image areas. The students should be able to explain this difference. These experiments are more costly to do, in the sense that specific lenses will have to be ordered from an optics catalog. Aberrations may be simply investigated by having the students rotate the lenses as they observe the images.

If time and motivation permit, a simple optical bench may be constructed to investigate the imaging equation. Modeling clay can be used to hold the surplus lenses alongside a meter stick. The source should be some interesting transparency or slide diffusely illuminated from the rear. The biggest obstacle in doing this experiment is that most students at this level may not yet have had any algebra. If they have had algebra, this experiment makes practical use of what they have learned. Simple Galilean and Keplerian telescopes may be constructed using this optical bench.

\section{H. Fresnel Lenses}

Another type of lens that students enjoy learning about is the Fresnel lens. It can be pointed out that the early use of Fresnel lenses was in lighthouses in Europe, as well as North America. If students have access to the World Wide Web, have them learn more about these large-scale lenses by connecting to the world wide web site at http://www.lib.utk.edu/lights.html.

\section{Microscopes}

A discussion of the simple microscope may or may not be within the scope or time frame of a unit on optics. If nothing else, obtain some simple 30X low-power pocket microscopes and have the students look at various objects. A particularly useful example is the pattern of colored dots found in color half-tone printing processes. This always leaves students surprised and amazed; the sensation of an apparent color is quite different from the colors of the actual dots!

\section{J. Optical Fiber}

One fascinating use of optics in modern technology is that of optical fibers. A simple discussion of how a fiber works may be given, once the concepts of refractive index and total internal reflection are introduced. Obtain some large-diameter plastic fiber for a whole variety of experiments. Small-diameter glass fiber is fine to show, but it is too difficult to couple enough light into the core for students to use. If the fiber is low- quality, it makes for a fun show in a dark room. Shine a bright light into one end (perhaps, but not necessarily a laser), and observe the scattered light that leaves the fiber at every micro-crack and defect. It should be pointed out that in fact, this is not a desirable characteristic of high-quality fiber! 
Use a card to block and unblock a flashlight placed at one end of the fiber, allowing Morse code to be sent. Students at the receiving end can attempt to decipher the message. Often, students ask if light of more than one color can travel at the same time in the fiber. Have them introduce light of different colors into opposite ends of a piece of fiber, to prove that light waves can indeed travel simultaneously in the same piece of fiber!

\section{WORKSHOPS AND PARTNERSHIPS FOR TEACHING OPTICS}

Workshop on optics are an effective resource for middle school teachers. We have given one workshop to introduce teachers to optics and how it may be taught. This workshop let the teachers do the same activities as the students. We have found that doing the activities is far more useful to the teachers than talking about the activities, and is also more fun. The reactions and feedback of these teachers has been instrumental in improving the activities.

A second activity we are involved in, as part of the Arizona Optics Industry Association, is in the training of teachers and volunteers in the optical community to work together in the schools. In many cases the optics-knowledgeable individual is a retired engineer or person active in applied optics. Our experience in this area is that a number of items must be considered for these kind of partnerships to be successful:

- The school district administration must be supportive of the program.

- The principal of the school must be supportive for it to succeed.

- The teachers must be self-chosen and willing participants.

- There is a need for a training and preparation phase. The teacher and engineer are paired up and allowed to develop an understanding of what each can bring to the partnership.

- The teacher must identify his or her needs in teaching optics.

- The relationship must be a long-term relationship. Very little is accomplished in one or two visits.

- Industrial partners and tours of industrial optics facilities are valuable. They are most valuable when the students have the proper preparation and the ground work has been laid.

\section{CONCLUSION}

There are a number of resources in the optics field for middle school teachers. We have identified a number of approaches that work, but still have much to learn. Since students at this age exhibit a great interest in optical phenomena, the teaching of optics is certainly an exciting and viable area.

\section{ACKNOWLEDGMENTS}

S. Pompea is an adjunct faculty member of Steward Observatory, University of Arizona and would like to acknowledge the university for use of its facilities. Both authors would like to thank the teachers and administration of Tucson Unified School District.

\section{REFERENCES}

M. J. Nofziger, "Optics curriculum for middle school students" Proceeding SPIE, this volume.

S. M. Pompea, editor, Great Ideas for Teaching Astronomy, 2nd edition, West Publishing Company, St. Paul, 1994. 